\title{
Research on Industrial Robot Teaching Pendant based on Android and its Realization
}

\author{
Yanan Yang, Feng Guo, Kaiji Han \\ (Tianjin Polytechnic University, School of electronic and Information Engineering, Tianjin, 300387, China)
}

\begin{abstract}
As the current industrial robots teaching systems have some disadvantages including high maintenance cost, poor portability and operational complexity, an industrial robot teaching system based on Android platform has been developed. The teaching pendant uses Android system equipment for industrial robot control terminal that the user is transmitted to the robot controller by operating the robot teaching will be achieved task command, the controller receives a corresponding command to control the movement of each axis of the robot to achieve each goal pose. The teach pendant can be programmed module, teaching language is used in the robot pose memory recording the coordinates of each target, and ultimately to run the program through the teach pendant control, teaching and playback functions. Teach and controllers communicate using $T C P$ and UDP protocols, network communication across platforms through the socket, and add functionality and extend the heartbeat of many single-threaded communication function in communication. Experimental results show that this teaching system has the advantages of stable operations, simple operations as well as good portability. It will have a broad prospect of application.
\end{abstract}

Keywords: Android; teach pendant; robot; Programming Module

\section{Introduction}

With the extensive application of industrial robots in all walks of life, industrial robots Industrial robots optional one member of the teaching performance have higher requirements. Robot teaching system is an important tool to achieve human-computer interaction, robot control system is an integral part. Teach Android system compared to the traditional teaching system has more obvious advantages by installing software can teach this device as a teaching system to use. The traditional robot teaching system can only be taught on a specific device, the popularity is low, so the teaching system based on the Android platform to a large extent reduce the cost and improve the teaching system teaching system flexibility .

\subsection{Software System Design}

Industrial robot system comprising: a robot teaching three parts, controllers, robot body. The robot body and the control cabinet is connected by a cable to form a set of facilities, generally provided by the manufacturers. Teach and control host through WiFi connected, constitute a control system for independent research and development unit. Teach is the Android device, user input commands through the operation Task robot teaching device through WiFi wireless network transmission to the robot controller, thereby controlling the robot body to complete the corresponding task. General structure of the system as shown in Figure 1.

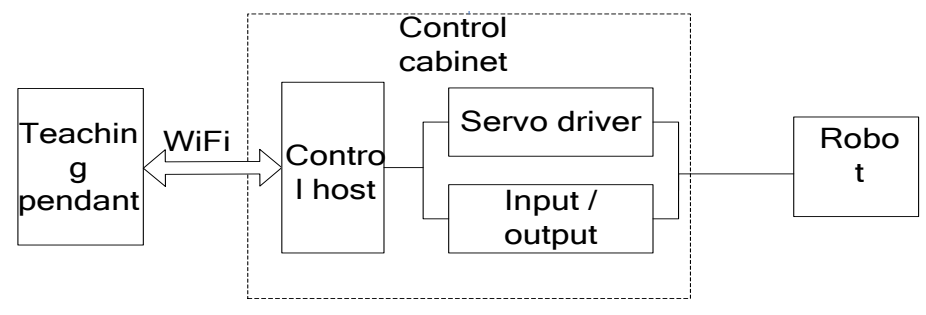

Fig.1 General structure of the system

The teach pendant and robot controllers connected to the same WiFi network, the controller sends UDP packets per second, three times, and the current state of motion parameter data broadcasting robots and the like. Android client via UDP packet capture and resolve to get IP address of the server to establish a TCP connection to get an IP address. Once connected, Android device can send commands to the server and receive status data of the robot controller receives commands and parse conversions robot control command to control the robot motion or change the operating parameters of the robot. Figure 2 is a flow chart of operation of the system 


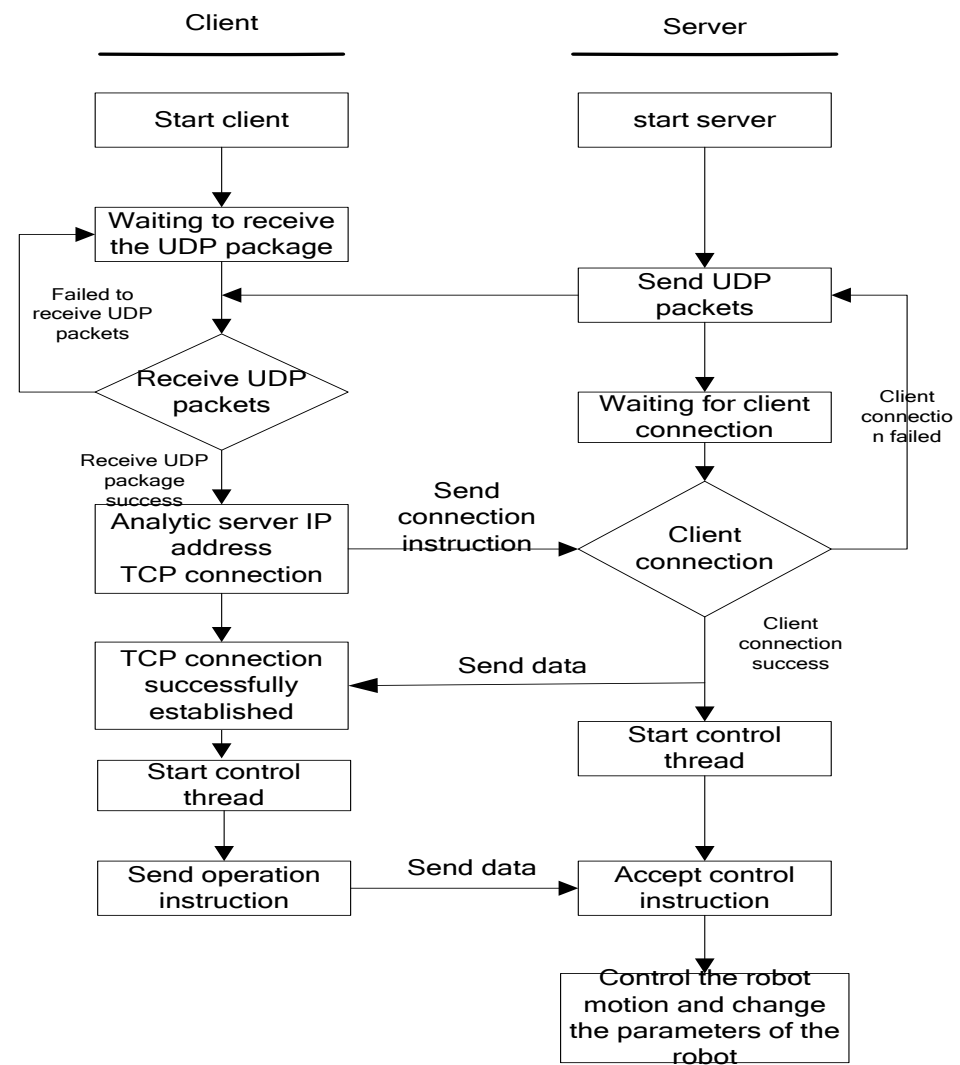

Fig.2 The flow chart of system work

\section{The main interface design}

The teach pendant main function is to implement a program of teaching the robot to find the origin, program editing, and program execution functions. When the user opens the software into the main interface shown in Figure 3. This interface, users can access important information by some robots top information bar icon above R, S, M represent the common state, state servo and motion state of the robot. Below is a privilege login button only when the user enters the correct password before you can use the Teach controlled movement of the robot. Below is a network connection button, teach with the robot controller via WiFi, the TCP / IP protocol to connect and communicate with each other. Thus, the connection interface, users can enter the address of the current robot controller is connected, when the robot successfully connected the button will display the corresponding robot controller information. The centerpiece is a pose is displayed parts in this section can display the current position of each axis of the robot and velocity information. The last button is a software switch button, exit the software when the button is clicked. The right of the interface is the teach function buttons, which is a program editing module, teaching modules and Looking for the origin.

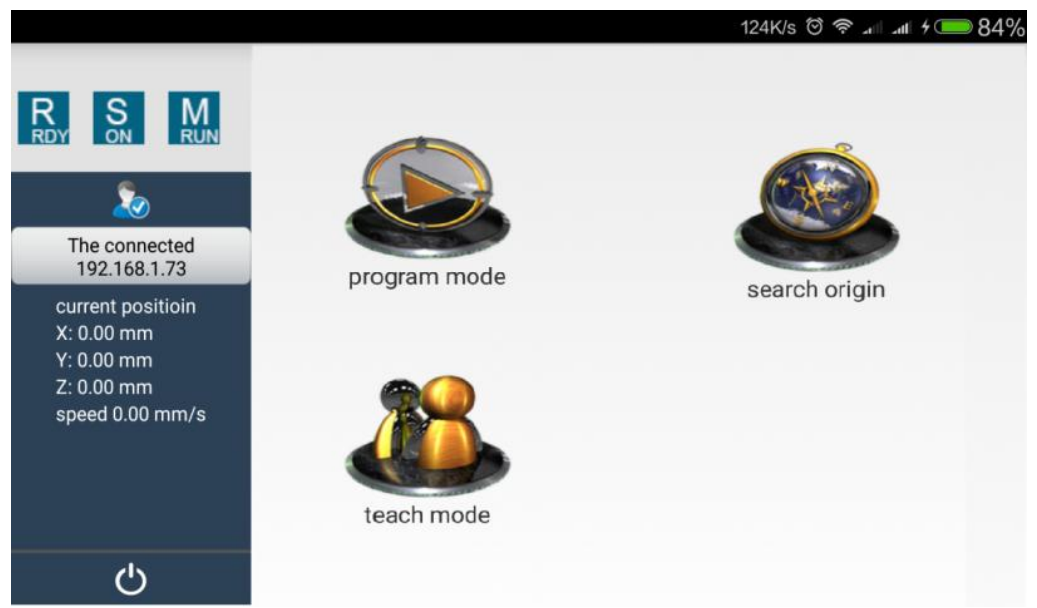

Fig.3 software interface 


\subsection{Looking for the origin}

When the initial use of the teaching aid to the robot operation need to find the origin, to determine the location of each axis of the robot. The machine boot finished, click "search for the origin of" button, the mechanical arm shaft automatically find the origin, the interface will pop up not cancel display dialog box prompts are looking for the origin, in the left can see coordinate axis in change, wait for the end of the origin and left servo axis will become 0 , is looking for the origin of the dialog will disappear and find the origin of the end.

\subsection{Manual teaching module}

In order to ensure the flexibility of the robot so that it can complete a variety of complex operations, designed robot programming language. The robot can be taught via teach programming, a control panel for the program to explain the job to achieve reproduction. Teach program throughout the entire process from the robot to teach reproduction, it plays a vital role. FlexPendant user manual teach-controlled robot to a particular location, the position coordinates of each robot axis write instruction; then, repeat the manual teaching and program instructions written until the completion of programming; Finally, teach program is downloaded to the next crew, auto-run program to teach reproducible teaching operation. Throughout the process, teaching mainly provides manual control interface for each axis and complete the program editing. Manual teaching interface shown in Figure 4. Radiobutton in the software can choose different axes of the robot through the top, through the positive and negative direction buttons to control the movement of the robot axes. On the right by setting the step size and speed buttons change the current movement of the robot step length and speed. Click into the programming button to enter the program edit screen.

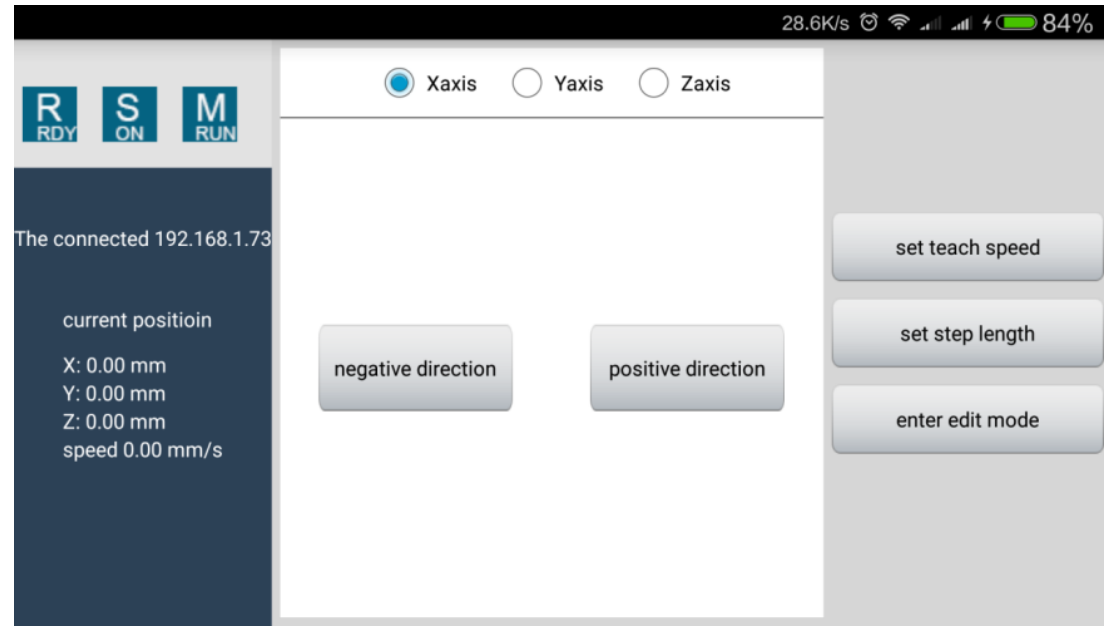

Fig.4 Manual teaching

\subsection{Program editing module}

Robot teaching and playback functions based teaching program for the carrier to achieve, so that the robot can teach the program flexibility to complete a variety of complex operations. When the user uses the teaching pendant can be taught, and the preparation of teaching program recording movement position of the robot, after teaching is completed, the teaching program is downloaded to the control panel, the control panel of the teach-in procedures for parsing and processing, and finally control actuator reproduce the teach-in procedure. To teach the program to support teaching and playback functions to achieve the process shown in Figure 5.

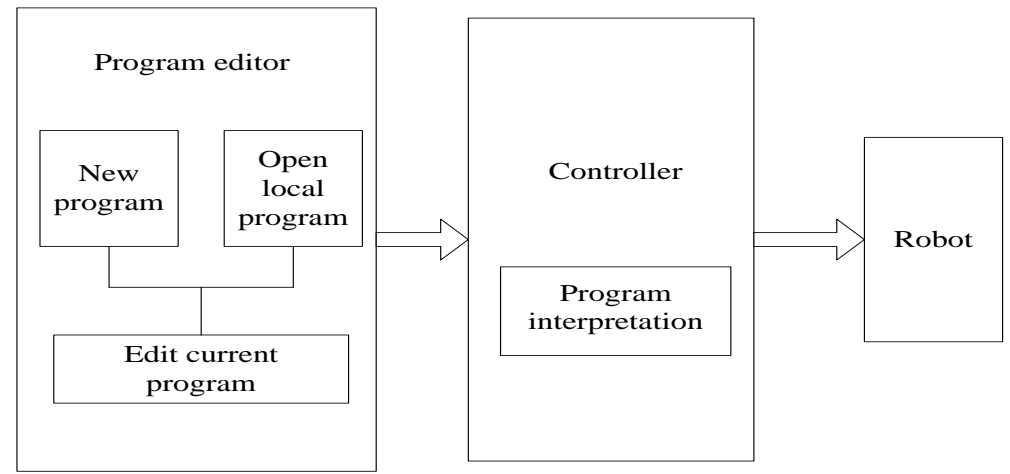

Fig.5 Program editing function realization process 
Program run settings menu bar, including the file menu, support for single step, single cycle, cycle execution, stop execution, download, return to the starting point. Program display column, the blue highlight line that the program runs, the line standard for program editing and state display switch. On the right side of the status bar, showing the input, output, running the current status of the data. Under the program operation mode, see the running state parameters; program edit mode, edit program. When in the program editing mode, the program will automatically switch to the program editor. When adding a line of instructions, you can choose the right hand to move, set the output, wait for input, the detection of the input tag to create a program command line. The software interface is shown in Figure 6

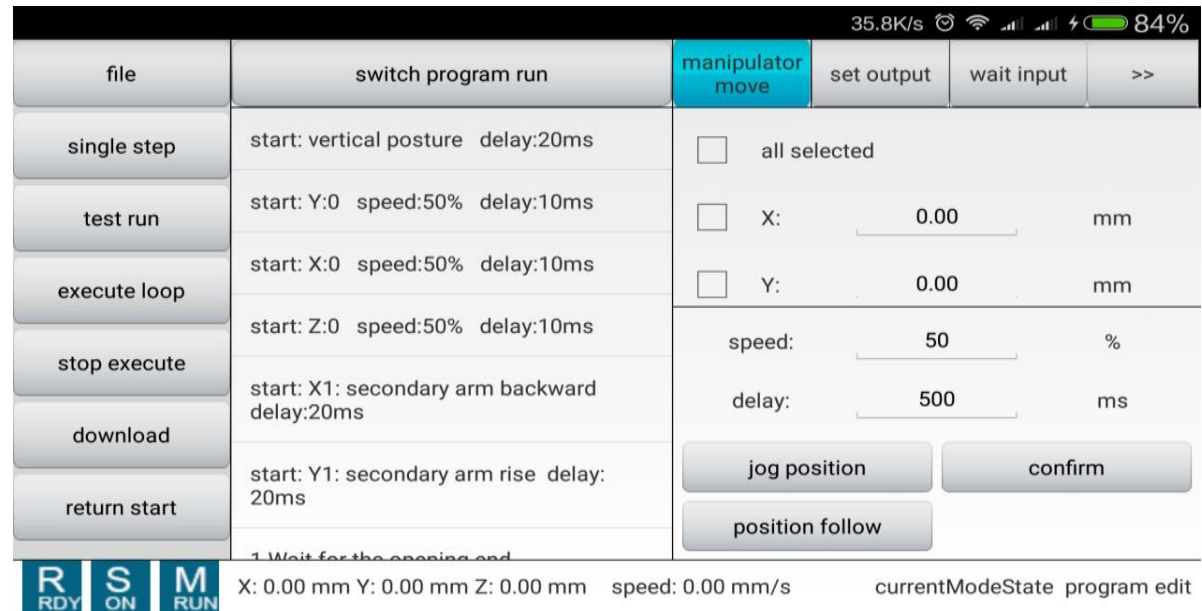

Fig.6 Program editing interface

\subsection{Communication module}

Teach-based software system implementation Android, Android system itself a response to the interface have been restricted, the interface within 5 seconds the system will not respond to the prompt message. Communication is a very time-consuming function module, in order not to affect the response time of the interface, communication can be run in the background, Android's Service components can run in the background. Teach software as Android system application software layer, the programming language used is Java, Java language provides encapsulation Socket classes to implement TCP client communications, provide encapsulation DatagramSocket classes to implement UDP communication client. So teach software directly using the Socket and DatagramSocket classes to implement TCP and UDP communications client communication client, respectively. Figure 7 shows the TCP Server and teaching is shown in TCP client communication processes.

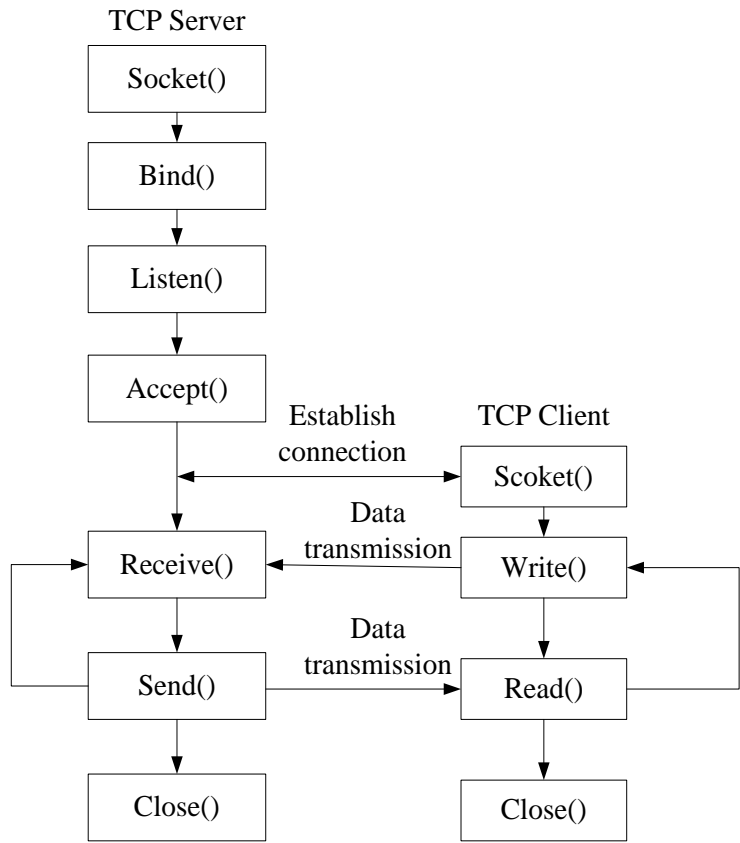

Fig.7 TCP Server and teaching is shown in TCP client communication process 
Control panel UDP client communication processes UDP server and teaching is shown in Figure 8. After controlling for a host server using datagram sockets to establish a communication server bound port number, UDP port number 3333 take. Not like the TCP communication as listening and waiting for a connection request, it can send data directly, and blocks reading. Teach uses the Java DatagramSocket establish communications client, using the connect function records the socket of the IP and port number, and send and receive data.

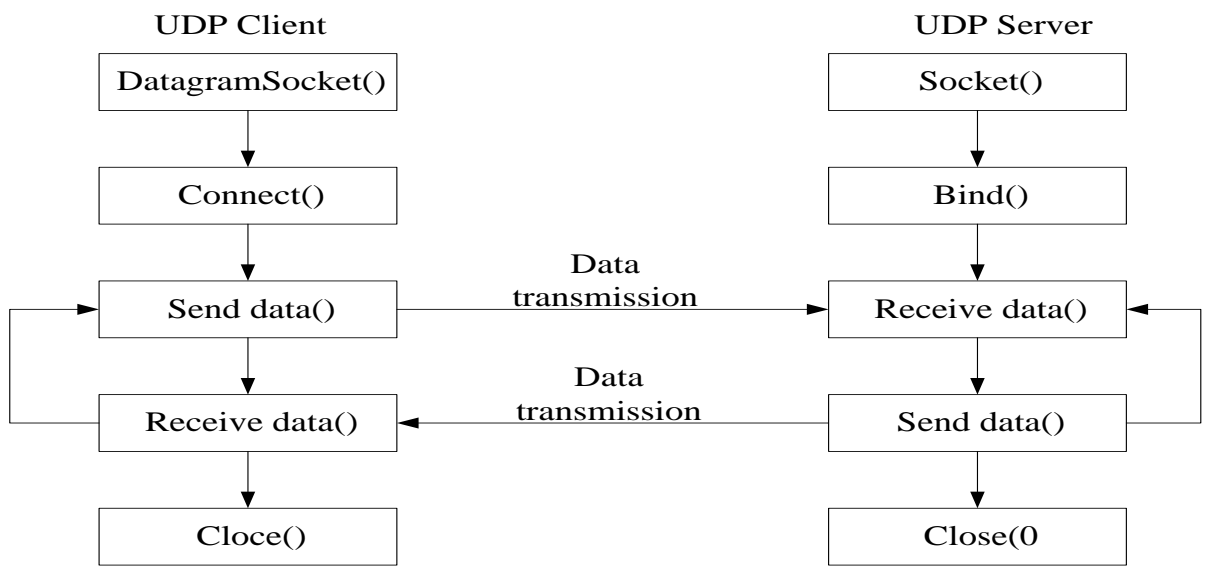

Fig.8 UDP server and the UDP client communication process

\section{Whole Test}

In an industrial robot in a laboratory experiment, the experiment shown in Figure 9 internet. Teach and control systems are connected to $\mathrm{WiFi}$, using the teaching pendant of the robot can accurately control the movement of the robot. During the experiment by changing the robot teaching system pose, speed and step length and other parameters, response and observation robot teaching system parameters display. The results of the surface of the teaching system can easily, quickly and accurately follow the instructions smooth robot motion.

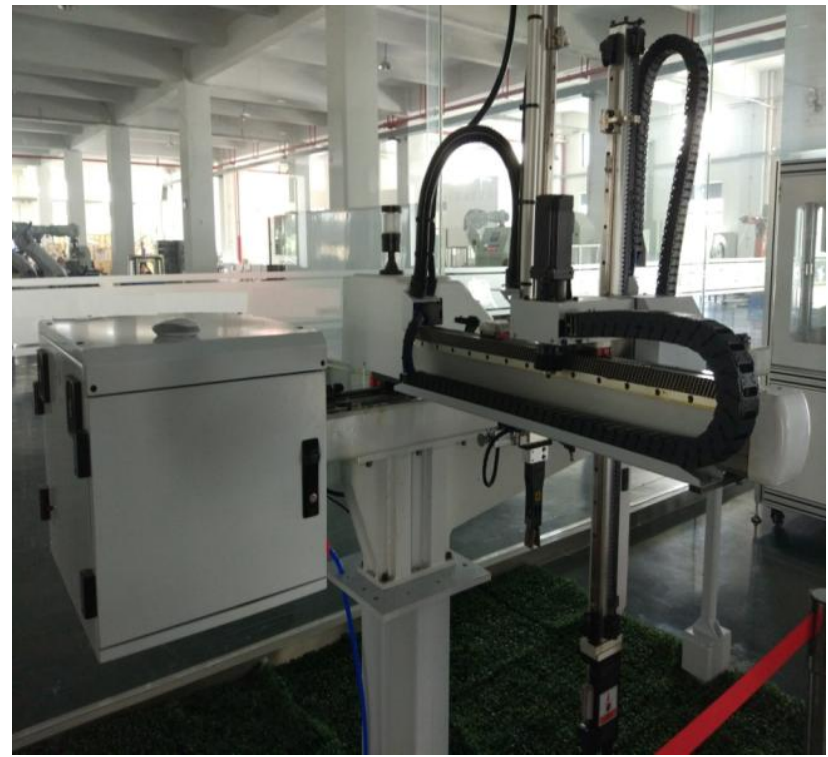

Fig.9 Experiment platform

\section{Conclusion}

From the experiment showed that the Android platform-based gesture control robot teaching system is accurate is simple, easy to carry, and low cost. Since the robot industry will be networked, intelligent development, mostly based teaching system Android platform is the future trend of development of the teaching system. Combining the Android programming, WiFi, information technology and numerical control technology, to achieve a multi-disciplinary, from the experimental point of view of the Android robot teaching system was exploratory study, from the technical level to prove the feasibility of the teaching system. 


\section{References}

[1] Jasin Amri, Alsaqour Raed, Abdelhaq Maha, et al. Review on Current Transport Layer Protocols for TCP/IP Model[J]. International journal of digital content technology and its applications, 2012,6(14):495.

[2] Suriyachai P, Roedig U, Scott A. A Survey of MAC Protocols for Mission-Critical Applications in Wireless Sensor Networks[J]. Communications Surveys \& Tutorials, 2012,14(2):240 264.

[3] Feng-shi Z. The Principle and Implementation of Heartbeat Packet based on Windows Socket Communication[J]. Journal of Shazhou Professional Institute of Technology, 2009,3:004.

[4] Asaf Shabtai, Yuval Fledel, Uri Kanonov, et al. Google Android: A Comprehensive Security Assessment[J]. Security \& Privacy, IEEE. 2010,8(2):35 44.

[5] Wei Tang, Guang Jin, Jiaming He, et al. Extending Android Security Enforcement with A Security Distance Model[A]. In Proceedings of the 2011 International Conference on Internet Technology and Applications. 2011, 08:1 4.

[6] Nonami K, Yuasa R, Waterman D, et al. Preliminary design and feasibility study of a 6-degree of freedom robot for excavation of un-exploded landmine. Autonomous Robots, 2005,18(3):293 301.

[7] Hasan A T, Ismail N, Hamouda A M S. Artificial neural network-based kinematics Jacobian solution for serial manipulator passing through singular configurations. Advances in Engineering Software, 2009, 41(2):359 367.

[8] Min-jae Oh, Sang-Moo Lee, Tae-wan Kim, et al. Design of a teaching pendant program for a mobile shipbuilding welding robot using a PDA[J]. Computer-Aided Design, 2010(3):172 182.

[9] Michael Franken. 24 INFORMA TIONSVERARBEITUNG: Wireless-Technologie[J]. Giesserei, 2007, 94(6):112 112

[10] He Renqing, Yan Gangfeng. Intuitive Control System for Industrial Robot Application Using Smartphone [J]. Applied Mechanics and Materials, 2014,685:284 288. 